Barreda Usó, Gemma.

Conservadora-Restauradora, Doctora en Bellas Artes.

Zalbidea Muñoz, Mㅁantonia.

Profesor Titular del Departamento de Conservación y Restauración de Bienes Culturales.

\title{
Tratamientos de consolidación de soporte rocoso con manifestaciones de arte rupestre. Abric de Pinos (Benissa- Alicante).
}

\section{Treatments of consolidation of rock support with rock art manifestations. Abric de Pinos (Benissa-Alicante).}

TIPO DE TRABAJO:

Comunicación.

PALABRAS CLAVE:

Consolidación, arte rupestre, soporte pétreo.

KEY WORDS:

Consolidation, rock art, stone support.

RESUMEN

El presente trabajo de investigación se ha centrado en el estudio comparativo entre diversos materiales consolidantes para su aplicación en soportes pétreos con manifestaciones de arte rupestre. Concretamente el testado de materiales se ha llevado a cabo sobre material rocoso procedente del Abric de Pinos (Benissa-Alacant), cuya problemática principal presenta una superficie descohesionada microscópicamente generando pulverulencia.

\section{ABSTRACT}

The present research work has focused on the comparative study between diverse consolidating materials for their application in stone supports with manifestations of rock art. Concretely the test of materials has been carried out on rocky material from Abric de Pinos (Benissa-Alacant), whose main problematic has a microscopically shredded surface generating pulverulence.

CONTENIDO.

INTRODUCCIÓN.

El presente trabajo de investigación muestra el estudio comparativo entre diversos materiales consolidantes para su aplicación en soportes rocosos con manifestaciones de arte rupestre, cuya superficie presenta descohesión microscópica en forma de pulverulencia. 
El proceso de consolidación es actualmente una de las actividades más importantes, ya que ésta es una de las fases determinantes del proceso de restauración. La consolidación, es considerada como un proceso activo y consistente en impregnar los materiales (en este caso pétreos), deteriorados con sustancias químicas penetrantes a fin de restablecer la cohesión, resistencia y firmeza que con el tiempo y los factores ambientales, se han ido perdiendo.

Los consolidantes son productos o sustancias cuya función es la de rellenar, los poros o los espacios vacíos de un sólido y devolver de este modo la resistencia mecánica o la estabilidad a los sólidos frágiles, impregnando capas y superficies friables, disgregadas, o pulverulentas.

Como restauradores, debemos ser conocedores de que un buen consolidante debe ser estable respecto a los agentes atmosféricos, la humedad y la luz, así como mostrar resistencia a la actividad biológica, y una toxicidad muy baja.

Las escasas investigaciones acerca del uso de tratamientos de consolidación y protección aplicados sobre material rupestre, junto con la gran variedad de productos de consolidación existentes en el mercado genera incertidumbres a la hora de elegir el tratamiento más adecuado para consolidar un material pétreo con manifestaciones rupestre. Con la experiencia adquirida, junto con el indispensable apoyo que hoy en día nos ofrecen los estudios científicos, ha dado lugar al descarte de alguno de estos productos y a la valoración de algunos de ellos, dando a conocer la complejidad de los materiales consolidantes y los aspectos tanto negativos como positivos que les caracterizan.

En el área de la conservación y restauración de material rupestre, se han utilizado diferentes tipos de productos consolidantes con la finalidad de frenar el estado de degradación y pulverulencia de las pinturas y los paneles. Si nos centramos en los productos más utilizados se destacan las resinas acrílicas.

La descohesión y pulverulencia es la alteración que afecta al Abric de Pinos, y por ello se analizan los diferentes consolidantes seleccionados, que aplicados a su soporte, y así poder determinar aquellos que son compatibles con la roca. Con el objetivo de obtener unos resultados que permitan establecer una comparativa entre los distintos productos y su interacción con la roca, fue necesario la elaboración de probetas que previamente fueron sometidas tanto a la acción de degradación medioambiental por causas naturales (depositándolas in situ), como aquellas, que fueron sometidas a diversos ensayos en laboratorio. De esta forma se observan y evalúan las propiedades de estos materiales, obteniendo resultados que permiten establecer mejoras en su uso y aplicación en futuras intervenciones.

A través de este estudio se constituyen premisas para el desarrollo y aplicación de consolidantes en el tratamiento de superficies con arte rupestre. Contribuyendo de este modo en la conservación de este patrimonio declarado Patrimonio de la Humanidad en 1998.

\section{ABRIC DE PINOS (BENISSA-ALICANTE).}

El conjunto pictórico rupestre del Abric de Pinos pertenece al término municipal de Benissa provincia de Alicante y está situado en un paraje pre-litoral (Ilustración 1), próximo al término municipal de Pinos ${ }^{1}$.
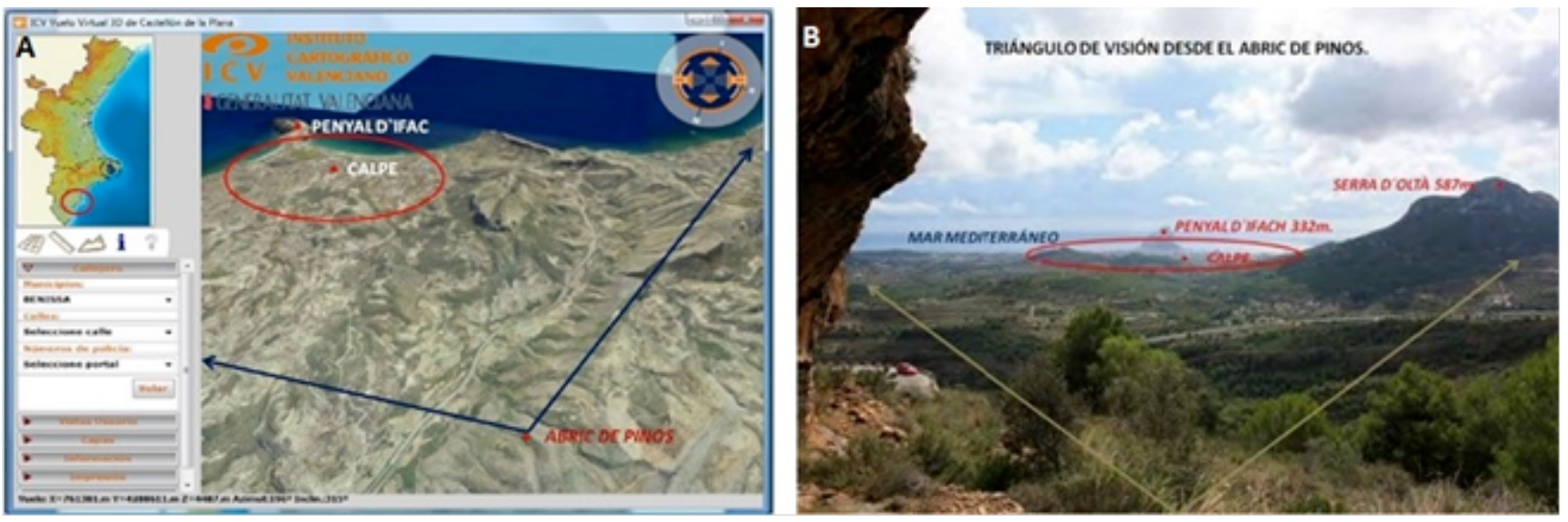

llustración 1.

${ }^{1}$ RONDA, Ana. Arqueología de Benissa. Instituto de Cultura "Juan Gil-Albert". 1990. pp. 13-23. 
Desde el punto de vista geológico, el término en el que se encuentra del Abric de Pinos pertenece al denominado Prebético. El abrigo rocoso sobre el que se hallan las pinturas está formado por materiales calizos de período Mesozoico (de sedimentación) que fueron elevados con la orogenia alpina en el periodo Terciario, alternándose estratos duros con otros más blandos. Como consecuencia, de esta alternancia de estratos, han dado lugar a la formación de cavidades (Ilustración 2.A), originadas a partir de un proceso de erosión por disolución, denominado Karstificación. Este fenómeno se ve favorecido por la presencia de fisuras, que se hacen en la roca permeable, facilitando la penetración del agua, que es el principal factor que interviene en la formación de las cavidades y por otros agentes de alteración físicos como erosión eólica.
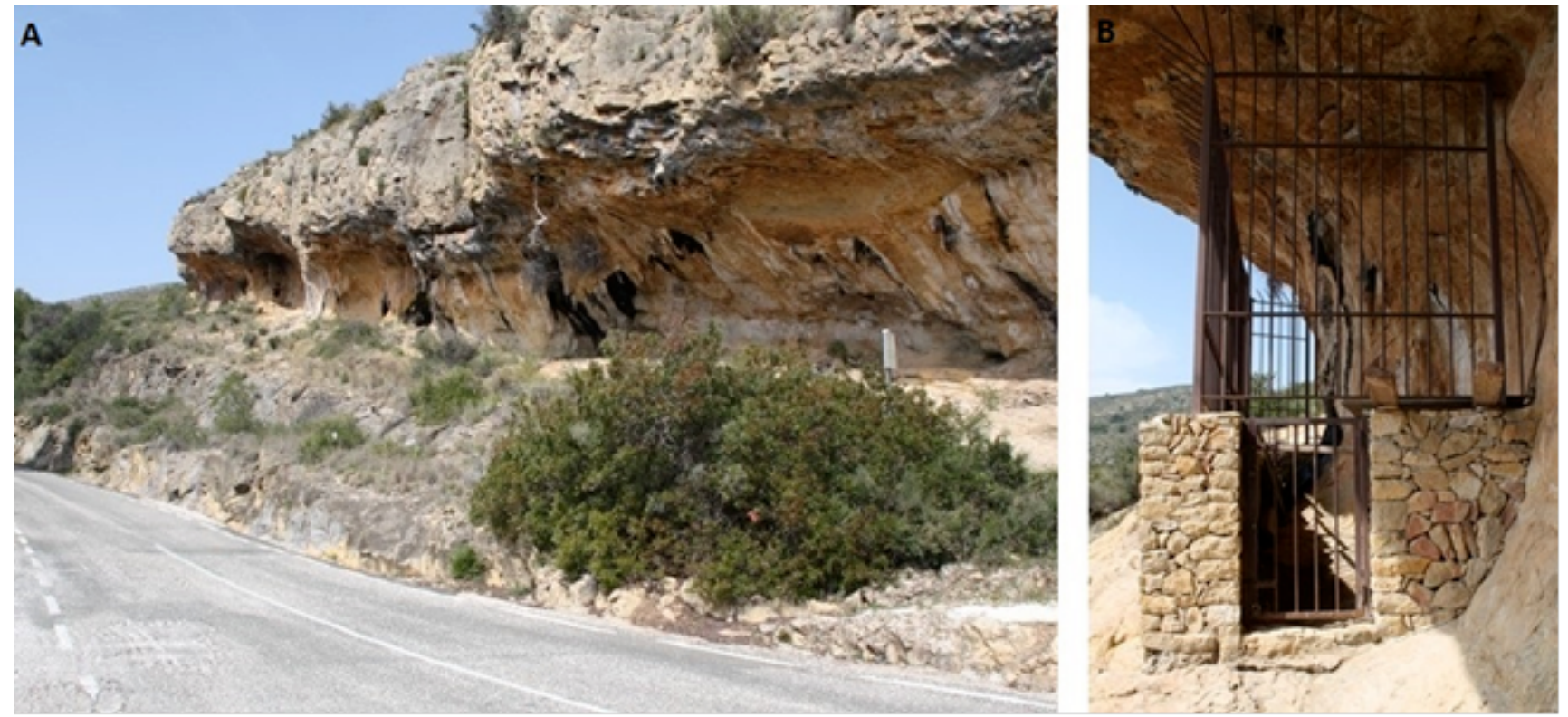

Ilustración 2.

Las pinturas rupestres del abrigo se encuentran representadas sobre un panel rocoso calizo que está en continuo proceso de evolución geológica natural, en consecuencia, se produce un envejecimiento natural del propio material constituyente imposible de frenar. Con los resultados de los estudios expuestos en este proyecto, se podría paliar estos procesos de alteración pero nunca erradicarlos.
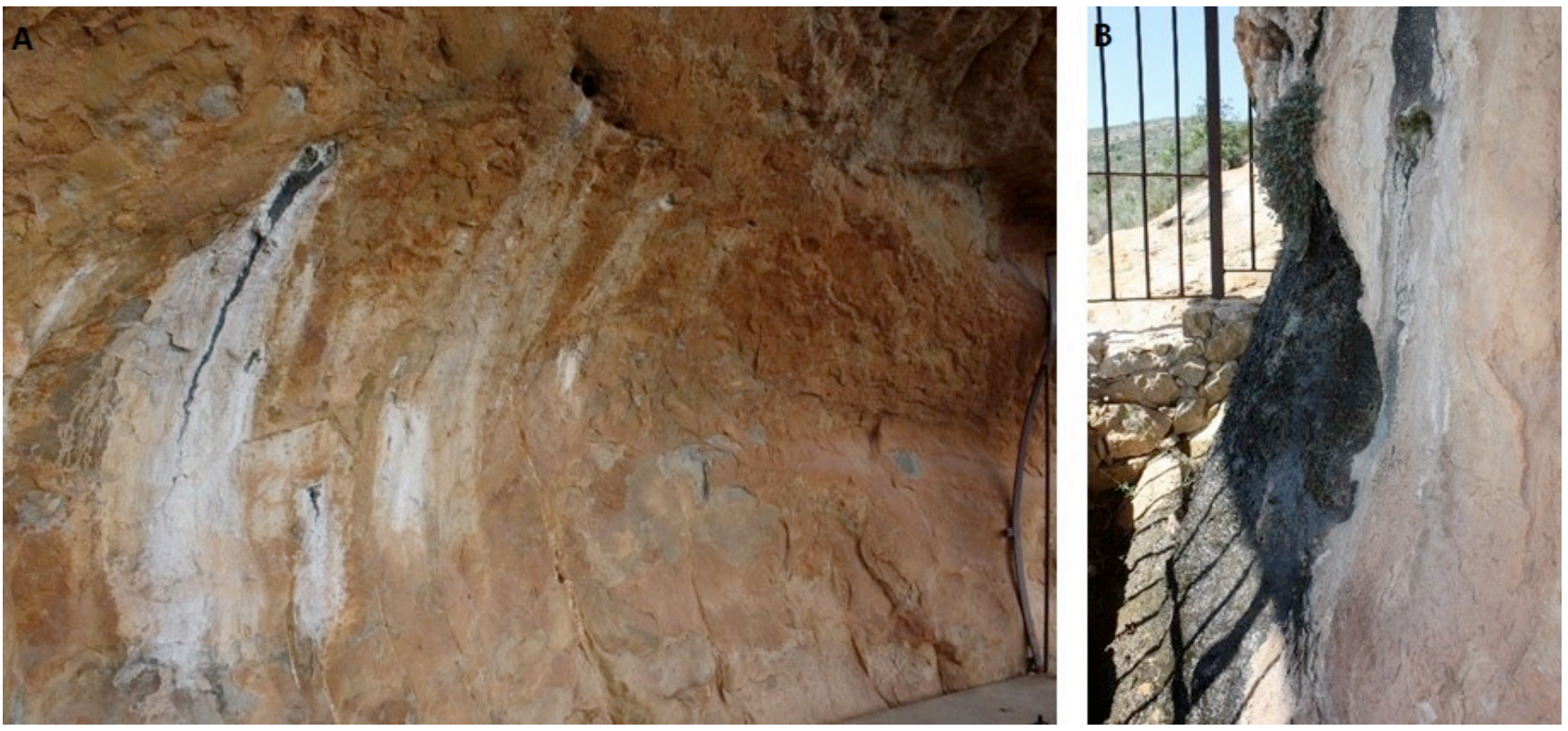

Ilustración 3. 
La cavidad rocosa en la que se ubican las pinturas, mide $5 \mathrm{~m}$ de profundidad por $7 \mathrm{~m}$ de altura. Éstas fueron descubiertas en 1970 , y se incluyen en el periodo estilístico denominado Arte Levantino, aunque en este abrigo existen además algunas representaciones que podrían ubicarse dentro del Arte Esquemático. Según los estudios realizados Mauro Hernández, Pere Ferrer y Enrique Català ${ }^{2}$, las pinturas representadas, se distribuyen en 4 paneles. Las figuras más representativas son; dos zoomorfos enfrentados en sus cuartos traseros y una figura humana femenina (llustración 4).
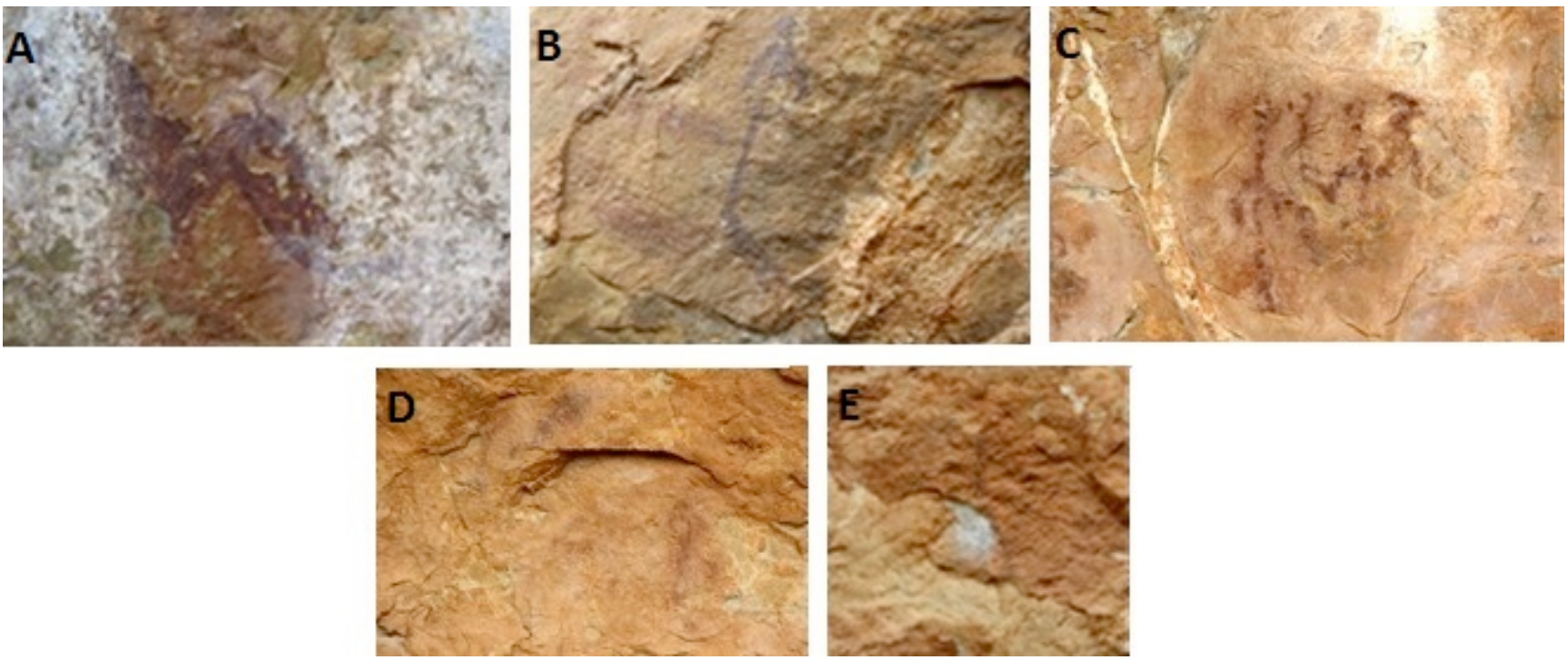

Ilustración 4.

El Abric de Pinos al igual que ocurre en la mayoría de enclaves con arte rupestre, por sus condiciones de exposición directa al aire libre, presenta degradación natural del soporte, fenómeno geológico que está en continua evolución y que es imposible detener, ya que no se pueden controlar las condiciones medioambientales a las que el abrigo está expuesto, tanto climáticas como geológicas. Los procesos de alteración natural debido a los efectos medioambientales y biológicos, ha provocado en el soporte cambios estructurales y de composición. A estos efectos naturales se añaden los daños antrópicos provocados por el hombre. Estos procesos se caracterizan por ser lentos, complejos e irreversibles.

Para detectar las afecciones que el material pétreo presenta se realizaron analíticas al microscopio electrónico de barrido (SEM-EDX) ${ }^{3}$, de este modo se obtuvo información de la composición química y la morfología estructural del material pétreo. En este caso se detectó que el material presentaba una delgada capa de sulfato de calcio en el interior y en la parte superior de la muestra. La presencia de sulfato cálcico en la capa más externa es debida a la sulfatación de la piedra caliza por efecto de los compuestos de azufre suspendidos en la atmósfera. Mientras que la capa de sulfato de calcio interior, puede ser debida a otro proceso de sulfatación producido con anterioridad y que con el tiempo ha sido recubierta por una nueva capa de carbonatación. La presencia del sulfato cálcico en el interior de la estructura pétrea es realmente preocupante debido a que esta formación constituye una capa mucho más friable e higroscópica que con el tiempo debilitará la zona provocando daños físico-químicos en el soporte.

Como apreciamos en la llustración 5.A., la cavidad presenta mayor grado de deterioro en la franja inferior que en la superior, donde se encuentran las pinturas. Esto es debido a que esta zona sufre mayores ciclos de insolación durante el año, ocasionando cambios bruscos de temperatura, que junto a la humedad relativa ambiental y el aporte de sales solubles, procedentes del mar, o del subsuelo. Estas sales, incluso pueden contenerse en la propia roca y emerger al infiltrar el agua de lluvia a través de la estructura pétrea. Generado así alteraciones físico-mecánicas en la roca originando daños por descohesión (Ilustración 5.B.) en forma de arenización, deplacado, fisuración, etc.

\footnotetext{
${ }^{2}$ HERNÁNDEZ, Mauro et al. Arte Rupestre en Alicante, 1988. pp. 312.

${ }^{3}$ Análisis científicos con microscopía electrónica de barrido acoplado a un sistema de microanálisis (SEM-EDX) y difracción de rayos X, realizados en el laboratorio de materiales del Institut Valencià de Conservació i Restauració de Béns Culturals.
} 

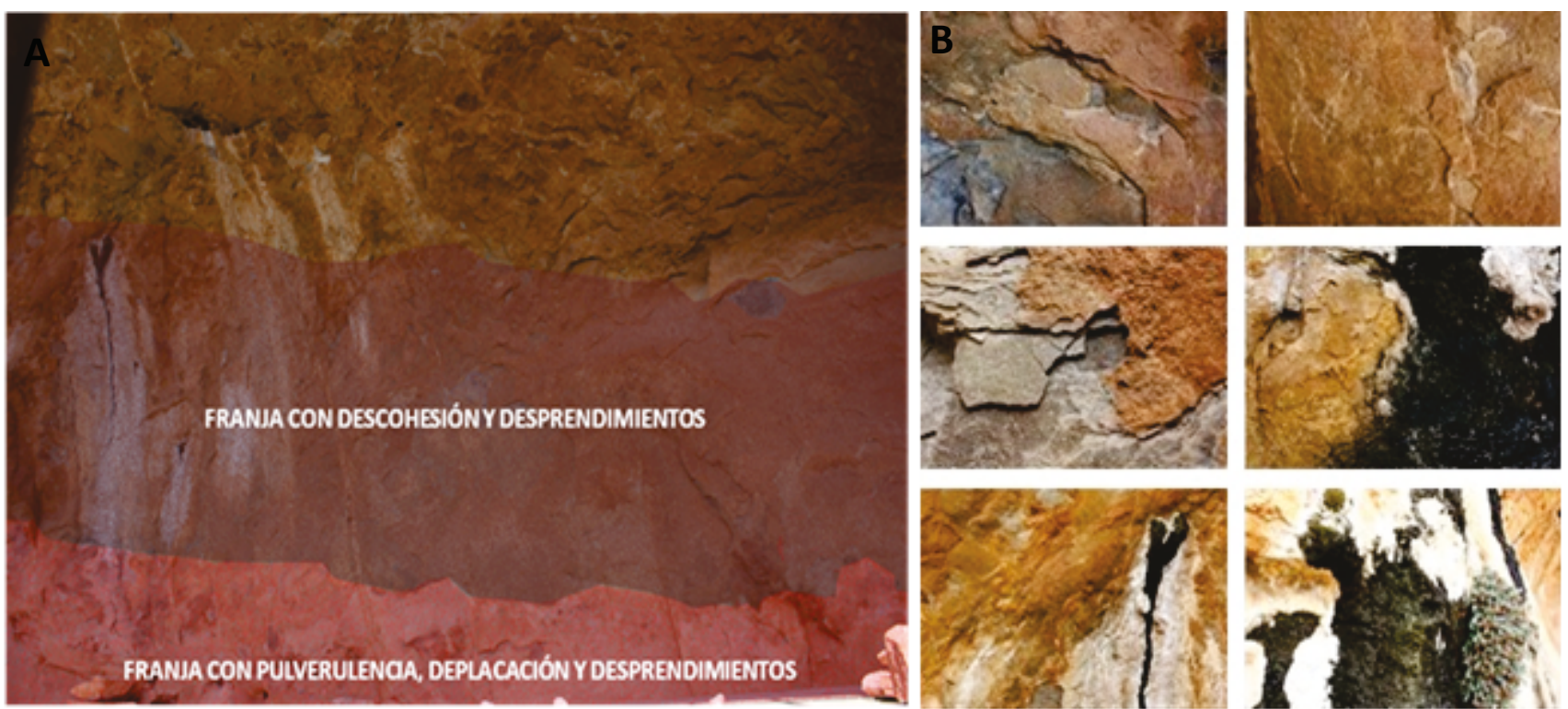

llustración 5.

Una vez analizado el estado de conservación que presenta el abrigo, se procedió a la clasificación de productos consolidantes que podrían solucionar la problemática de descohesión que presenta el soporte. El objetivo principal es comparar la efectividad de ciertos polímeros sintéticos utilizados ${ }^{4}$ en anteriores ocasiones en otros abrigos con manifestaciones de arte rupestre. Estos materiales son: elastómeros fluorados (Fluoline $\mathrm{CP}^{\circledR}$ ), polímeros acrílicos (Paraloid B-72 ${ }^{\circledR}$ ), micro emulsión acrílica (Acril ME ${ }^{\circledR}$ ) y un material inorgánico nano particulado, como el Nanorestore ${ }^{\circledR}$, hasta la fecha no utilizado en este campo. Para probar su eficacia, fue necesaria la elaboración de probetas realizadas con soporte pétreo procedente de los alrededores cercanos al abrigo. Posteriormente, estas probetas, se sometieron a distintos ensayos tanto en laboratorio, como a alteración natural depositándolas in situ.

Hasta la actualidad, en manifestaciones de arte rupestre uno de los productos más utilizados en consolidación puntuales o de emergencia, ha sido el polímero Paraloid B- $72^{\circledR}$, su uso viene derivado de otros campos de conocimiento afines al arte rupestre como la pintura mural y los monumentos pétreos, consiguiendo gran aceptación en el campo de la restauración por su fácil uso, aplicación y buen comportamiento. Si se realiza una revisión bibliográfica exhaustiva del uso de este material, se encuentran numerosos defensores y detractores, es más, no solo del producto en sí, sino de las condiciones de uso y aplicación. En cuanto al arte rupestre y al arte parietal prehistórico son pocos los estudios realizados sobre el uso y aplicación de este material, aún así, su uso y aplicación se ha visto justificado en este ámbito por la carencia tanto de estudios como de materiales aplicables a tales menesteres.

La micro emulsión Acril $\mathrm{ME}^{\oplus}$, tiene unas reducidas dimensiones de las partículas (alrededor de 50 micrón). Le proporcionan una baja viscosidad y una mayor capacidad de penetración en substratos porosos con respecto a las normales emulsiones acrílicas. Además tiene un bajo impacto cromático y excepcional resistencia al amarilleamiento, buena estabilidad mecánica y transparencia. En este estudio, fue utilizado en un porcentaje de 1:4 con agua desmineralizada y un 10\% de alcohol etílico para favorecer la penetración.

En cuanto al Fluoline $\mathrm{CP}^{\circledR}$, se trata de una de las resinas de nueva generación a base de fluoroelastómeros y polímeros acrílicos disueltos en acetona. De fácil aplicación, su elevada resistencia a los agentes atmosféricos, no genera variaciones cromáticas debido a su resistencia a los rayos UV. Estas características, lo hacen apto para tratamientos al aire libre con problemas de descohesión. En este caso se utilizó sin diluir, como indican las referencias técnicas del comerciante.

Finalmente se realizaron probetas con Nanorestore ${ }^{\circledast}$, ideal para consolidar piedras con matriz carbónica. Constituido por partículas nano-estructuradas de cal apagada dispersas en alcohol isopropílico que garantiza una óptima penetración en la matriz porosa, quedando las nanopartículas de hidróxido cálcico insertadas en los intersticios y en la red porosa a consolidar. Éstas, por acción del anhídrido carbónico atmosférico se transforman en carbonato de calcio originando una red de micro-cristales de calcita que confieren elevadas propiedades mecánicas al soporte, sin introducir materiales extraños a la naturaleza química de la superficie tratada. Se trata

\footnotetext{
${ }^{4}$ Las resinas termoplásticas como son las acrílicas, han sido ampliamente utilizadas desde mediados del siglo XX, aunque su uso ha ido variando con los años. Dentro del campo de los polímeros acrílicos los más empleados para la consolidación de materiales disgregados son el Paraloid B-72.
} 
Barreda Usó, Gemma. Zalbidea Muñoz, Mạ Antonia

Tratamientos de consolidación de soporte rocoso con manifestaciones de arte rupestre. Abric de Pinos (Benissa-Alicante)

III CONGRESO INTERNACIONAL DE INVESTIGACIÓN EN ARTES VISUALES :: ANIAV 2017 :: GLOCAL [codificar, mediar, transformar, vivir] http://dx.doi.org/10.4995/ANIAV.2017.5861

por lo tanto de un material cuya composición química es totalmente afín y compatible a la del soporte de roca original. En este estudio se utilizó directamente con una porcentual del $10 \%$ de alcohol isopropílico desnaturalizado para mejorar la penetración.

\begin{tabular}{|c|c|c|c|c|}
\hline PRODUCTO & PROPIEDADES & COMPOSICIÓN & DILUYENTE & $\%$ \\
\hline Paraloid B-72 ${ }^{\circ}$ & Consolidación, fijación y adhesión & Metilacrilato y etilmetacrilato & Acetona & $3 \%$ \\
\hline Acril ME ${ }^{\circ}$ & Consolidación. & Micro emulsión acrílica. & Agua desmineralizada & $1: 4$ \\
\hline Fluoline CP & $\begin{array}{l}\text { Pre consolidación, consolidación y } \\
\text { protección. }\end{array}$ & $\begin{array}{c}\text { Fluoroelastómeros y polímeros } \\
\text { acrílicos. }\end{array}$ & Acetona & \\
\hline Nanorestore ${ }^{\circ}$ & Consolidación. & Hidróxido de calcio. & $\begin{array}{l}\text { Agua desmineralizada } \\
\text { Alcohol isopropílico }\end{array}$ & \\
\hline
\end{tabular}

Tabla 1.

Una vez obtenida la caracterización y composición de la piedra y seleccionados los productos, procedimos a la realización estandarizada de las probetas que someteremos posteriormente:

1. Ensayo de absorción de agua por capilaridad UNE-EN 15801, (Determinación de la absorción de agua por capilaridad).

2. Ensayo de cristalización de sales: recomendado por la normativa RILEM33 1980 (Ensayo no V. 1b).

2.1. - Por inmersión total siguiendo los ciclos de cristalización.

2.2. - Por inmersión y secado libre.

3. Ensayo de envejecimiento acelerado con variaciones de temperatura, humedad y radiación UV, siguiendo las pautas marcadas por ASTM Internacional en el ensayo D1183-03.

4. Ensayo de la penetración del consolidante por tinción.

5. Estudio colorimétrico, siguiendo la normativa UNE-EN 15886 (Medición de color de superficies).

\section{CONCLUSIONES.}

Los resultados comprobados tras las observaciones con SEM-RDX de las probetas antes y después de la aplicación del consolidante, se determina que el Paraloid B-72 ${ }^{\circledR}$ provoca acumulación excesiva de resina en determinadas zonas reduciendo la permeabilidad al vapor de agua (Ilustración 6). Éstas con el tiempo envejecerán de forma diferente al resto de la superficie que queda más porosa. Al igual que el comportamiento mecánico también será diferencial creando tensiones en el interior del material con el peligro de formación de fracturas y desprendimientos. El aspecto cromático también se verá afectado ya que las zonas con acumulaciones muestran un aspecto plastificado.

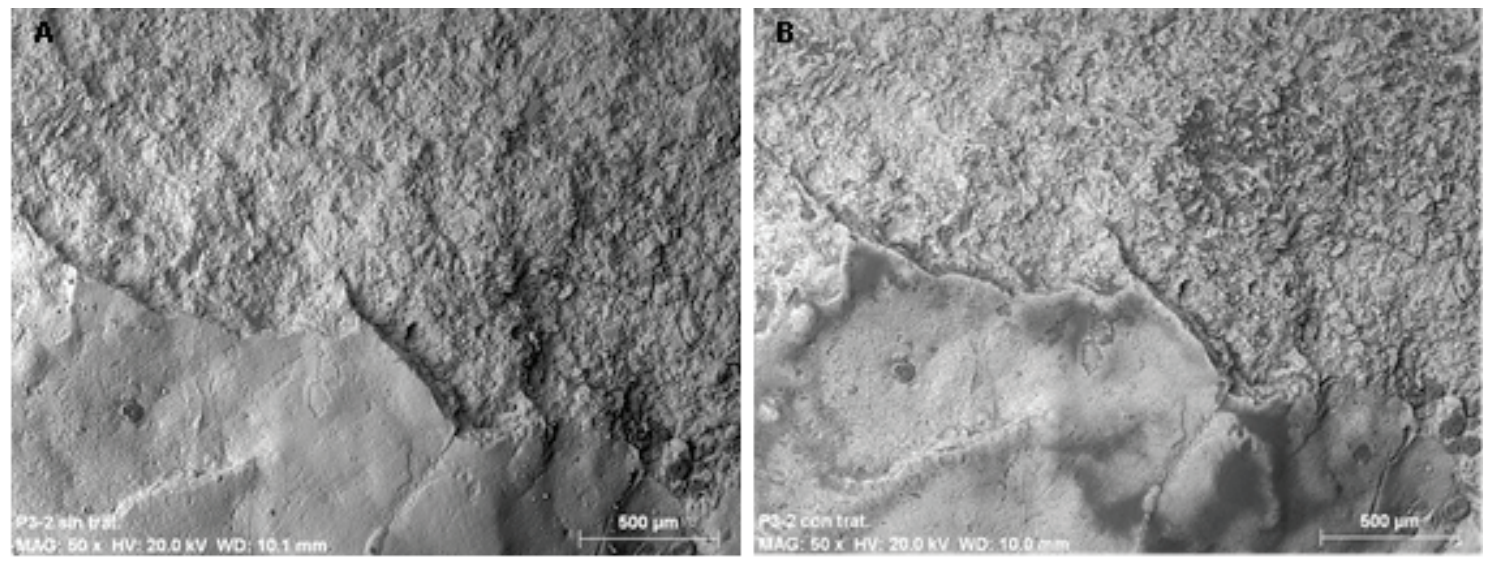

llustración 6. 
Barreda Usó, Gemma. Zalbidea Muñoz, Mạ Antonia

Tratamientos de consolidación de soporte rocoso con manifestaciones de arte rupestre. Abric de Pinos (Benissa-Alicante) III CONGRESO INTERNACIONAL DE INVESTIGACIÓN EN ARTES VISUALES :: ANIAV 2017 :: GLOCAL [codificar, mediar, transformar, vivir] http://dx.doi.org/10.4995/ANIAV.2017.5861

Con respecto al Acril $\mathrm{ME}^{\circledR}$ (Ilustración 7) se observa la formación de una capa superficial más homogénea con respecto al Paraloid B$72^{\circledR}$, pero también reduce de forma importante la permeabilidad al vapor de agua. La resina se deposita en las zonas cóncavas y en las partes más prominentes apenas se acumula. Esta distribución poco uniforme creará envejecimiento irregular, daños mecánicos y la posibilidad de formación de subproductos debido al carácter orgánico del producto. Los subproductos interaccionan con la roca degradándola, además de favorecer el crecimiento de microorganismos. En cuanto a las variaciones cromáticas afecta a la superficie mostrando un aspecto plastificado.

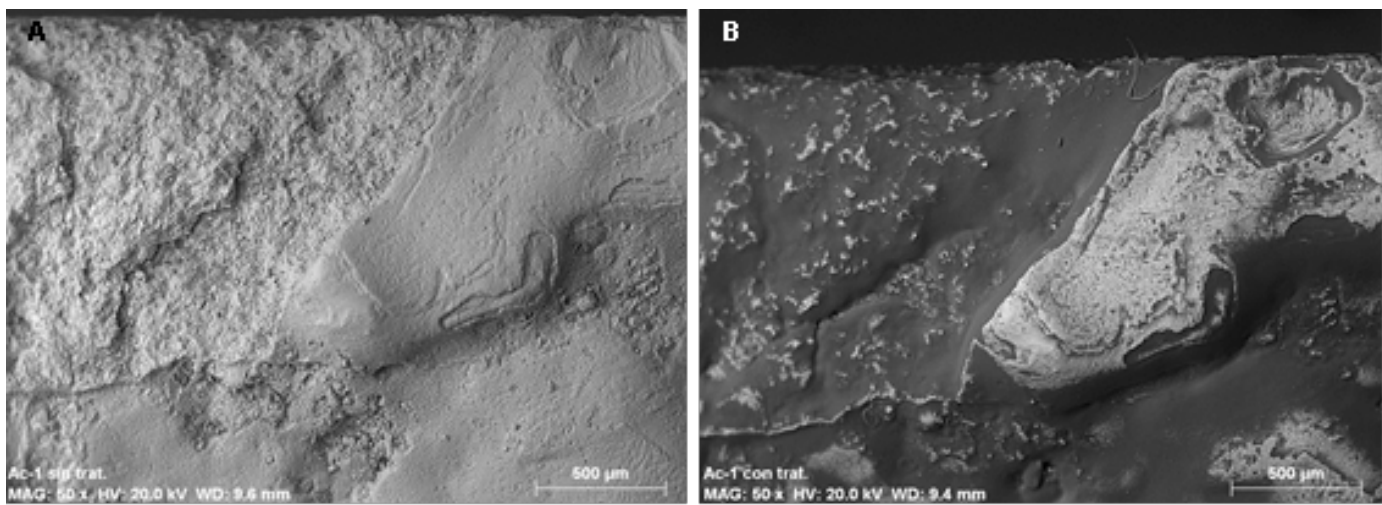

llustración 7.

Con el Fluoline $\mathrm{CP}^{\circledR}$ se observan acumulaciones superficiales pero sin llegar a formar un film compacto, es decir, respeta la porosidad pétrea, al contrario de lo que ocurre con el Paraloid B- $72^{\circledR}$ y el Acril ME ${ }^{\circledR}$.

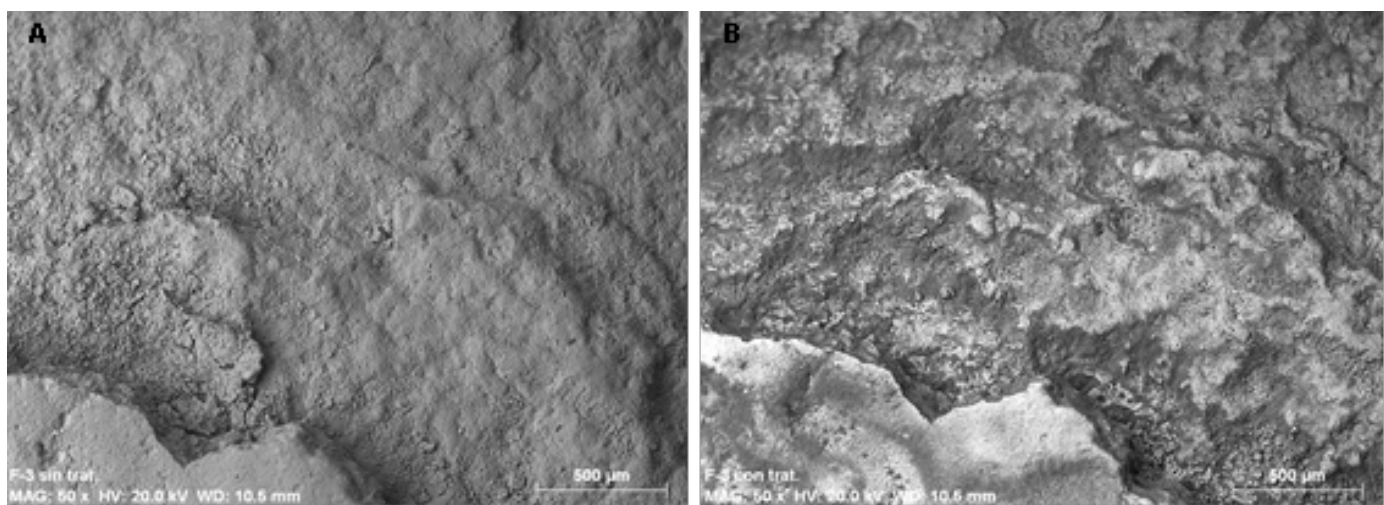

Ilustración 8.

Finalmente con el Nanorestore ${ }^{\circledR}$ (Ilustración 9) no se aprecian residuos de disolventes, no afecta el aspecto cromático de la roca, no genera films, ni afecta a la porosidad de la roca, es el producto más compatible con la naturaleza del soporte. Evitando de este modo posibles reacciones de los productos no aconsejados.
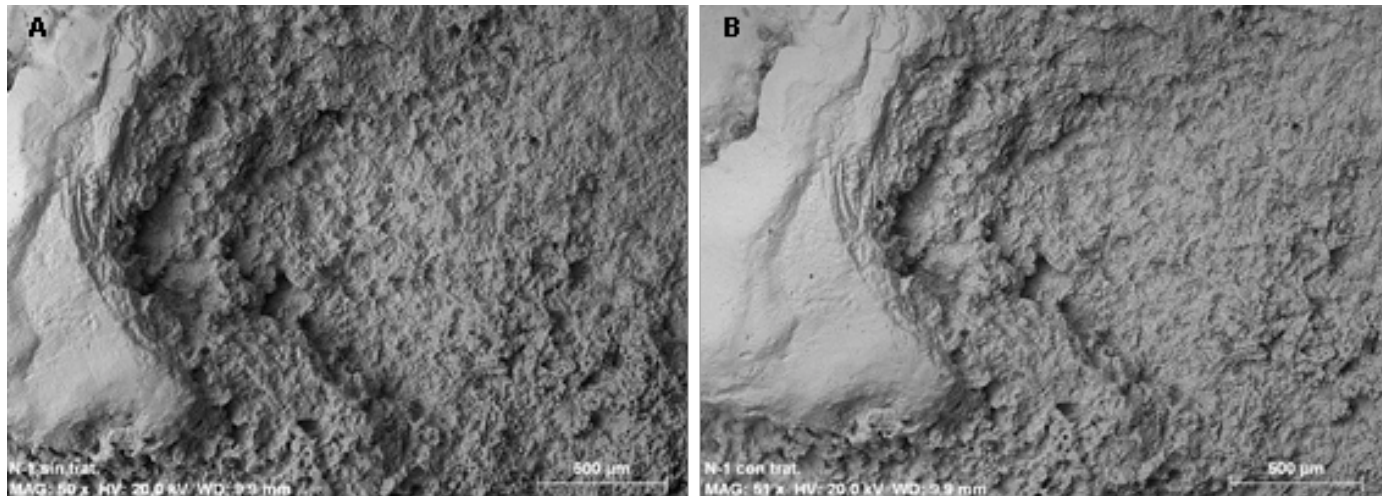

Ilustración 9. 
Barreda Usó, Gemma. Zalbidea Muñoz, Mạ Antonia

Tratamientos de consolidación de soporte rocoso con manifestaciones de arte rupestre. Abric de Pinos (Benissa-Alicante)

III CONGRESO INTERNACIONAL DE INVESTIGACIÓN EN ARTES VISUALES :: ANIAV 2017 :: GLOCAL [codificar, mediar, transformar, vivir] http://dx.doi.org/10.4995/ANIAV.2017.5861

Optaríamos por el uso de Nanorestore ${ }^{\circledR}$ como consolidante en el caso específico del Abric de Pinos, por tratarse de un producto de origen inorgánico que desempeña correctamente las exigencias necesarias de afinidad con el soporte, evitando de este modo aportar elementos de naturaleza diversa que con el tiempo ocasionan problemas por incompatibilidad de materiales. Además, se ha observado que el Nanorestore ${ }^{\circledR}$ no afecta a la capilaridad de la roca garantizando una buena permeabilidad al vapor de agua, ni genera cambios cromáticos apreciables, ni crea films superficiales compactos, manteniendo superficie estable y uniforme tras los ciclos de cristalización de sales. Cabe señalar que con el Nanorestore ${ }^{\circledR}$ solucionaríamos los problemas de descohesión, sin embargo sería necesaria una investigación paralela para determinar qué tipo de materiales serían óptimos para conseguir la adhesión del deplacado de la roca.

\section{FUENTES REFERENCIALES.}

AENOR. Asociación Española de Normalización y Certificación. Métodos de ensayo para piedra natural. Determinación del coeficiente de absorción de agua por capilaridad. UNE-EN 1925. 1999.; Conservación del patrimonio cultural. Método de ensayo. Determinación de la absorción de agua por capilaridad. UNE-EN 15801. 2010.; Conservación del patrimonio cultural. Método de ensayo. Medición de color de superficies. UNE-EN 15886. Madrid: 2011.

ASTM Internacional. Standart Practices for Resistance of Adhesives to Cyclic Laboratory Aging Conditions. D1183-03 United States: ASTM, 2003.

BALLESTER, Laura. Conservación de las pinturas rupestres del Levante Español. Tesis doctoral inédita. Universidad Politécnica de Valencia UPV. Departamento de Conservación y Restauración de Bienes Culturales. Valencia, 2003.

BELTRÁN, Antonio. Arte Rupestre Levantino. En: CAESARAUGUSTA no. 47-48. PSANA. Zaragoza: Editado por la Institución "Fernando el Católico". Excma. Diputación de Zaragoza, 1979. ISSN 0007-9502.

BENAVENTE, David et al. Estudio de propiedades físicas de las rocas. En: AEPECT. Enseñanza de las Ciencias de la Tierra. Girona: Editorial de la Universidad de Girona. Vol. 12, no. 1. Girona, 2004. ISSN 1132-9157.

HERNÁNDEZ Mauro et al. Arte Rupestre en Alicante. Alicante, 1998.

ICV. Instituto Cartográfico Valenciano [en línea]. [Citado enero 20, 2017]. Disponible en Web: http://www.icv.gva.es.

RILEM. Reunión Internacional de Laboratorios de Ensayo y de Investigación para Materiales de construcción. Pruebas recomendadas para medir el deterioro de la piedra y para evaluar la eficacia de los métodos de tratamiento. Ensayo no. V. 1b. París: RILEM Publicaciones SARL, 1980.

\section{ILUSTRACIONES.}

Ilustración 1. A) Visión virtual en 3D de la posición geográfica del abrigo. ICV. Instituto Cartográfico Valenciano [en línea]. [citado enero 20, 2011]. Disponible en Web: http://www.icv.gva.es. B) Triángulo de visión desde el abrigo.

Ilustración 2. A) Abric de Pinos, formado por fenómenos de Karstificación. B) Detalle del cerramiento del abrigo.

Ilustración 3. A) dentro del abrigo. B) desde el interior del abrigo, una vista de la entrada, la cancela y un fuerte y activo crecimiento biológico.

Ilustración 4. A) Cuartos traseros de dos zoomorfos de estilo levantino. B) Figura femenina estilizada de estilo levantino. D) Figuras de estilo esquemático. D) Restos de pigmento rojo. E) Motivo en forma de $\mathrm{Y}$ invertida que se conserva parcialmente.

Ilustración 5. A) Vista general del abrigo donde se observa como la parte inferior sufre mayor grado de insolación y deterioro, coladas activas que han generado cristalizaciones de carbonato cálcico. B) Detalle de alteraciones que sufre el soporte.

ANIAV Asociación Nacional de Investigación en Artes Visuales

This work is licensed under a Creative Commons Attribution-NonCommercial-NoDerivatives 4.0 International License (CC BY-NC-ND 4.0) 
Barreda Usó, Gemma. Zalbidea Muñoz, Mạ Antonia

Tratamientos de consolidación de soporte rocoso con manifestaciones de arte rupestre. Abric de Pinos (Benissa-Alicante)

III CONGRESO INTERNACIONAL DE INVESTIGACIÓN EN ARTES VISUALES :: ANIAV 2017 :: GLOCAL [codificar, mediar, transformar, vivir] http://dx.doi.org/10.4995/ANIAV.2017.5861

Ilustración 6. SEM-EDX. A) Probeta sin tratar. B) Probeta con dos capas de Paraloid B-72 ${ }^{\circledR}$ al $3 \%$ en acetona aplicado a pincel.

Ilustración 7. SEM-EDX. A) Probeta sin tratar. B) Probeta tratada con dos capas de Acril ME ${ }^{\circledR}$ al 1:4 en agua desmineralizada aplicado a pincel.

Ilustración 8. SEM-EDX. A) Probeta sin tratar. B) Probeta tratada con dos capas de Fluoline CP ${ }^{\circledR}$ aplicado directamente sin diluir.

Ilustración 9. SEM-EDX. A) Probeta sin tratar. B) Probeta con dos capas de Nanorestore ${ }^{\circledR}$ aplicado a pincel directamente sin diluir.

Tabla 1. Esquema de los productos y proporciones utilizados en esta investigación. 